\title{
Dimerization induced by C-terminal 14-3-3 binding is sufficient for BRAF kinase activation
}

Nicholas P.D. Liau ${ }^{1}$, Avinashnarayan Venkatanarayan², John G. Quinn ${ }^{3}$, Wilson Phung ${ }^{4}$, Shiva Malek², Sarah G. Hymowitz ${ }^{1}$, Jawahar Sudhamsu1,2*

${ }^{1}$ Department of Structural Biology, ${ }^{2}$ Department of Discovery Oncology, ${ }^{3}$ Department of Biochemical and Cellular Pharmacology, ${ }^{4}$ Department of Microchemistry, Proteomics and Lipidomics

Genentech Inc., 1 DNA Way, South San Francisco, CA 94080

*Corresponding Author, Email: jawahars@gene.com; Phone: 650-467-8221

\section{Supporting Information}


A

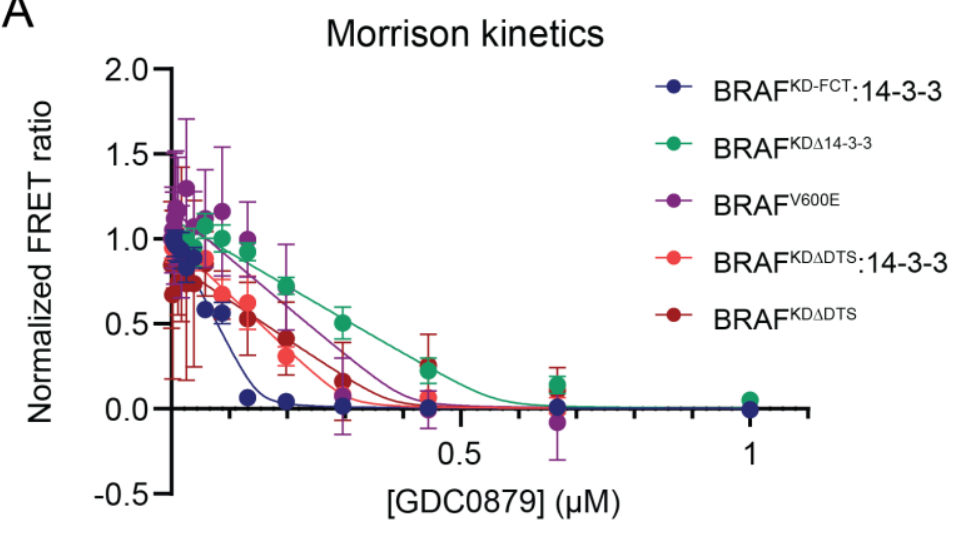

C TR-FRET ratio vs [PMEK] standard curve

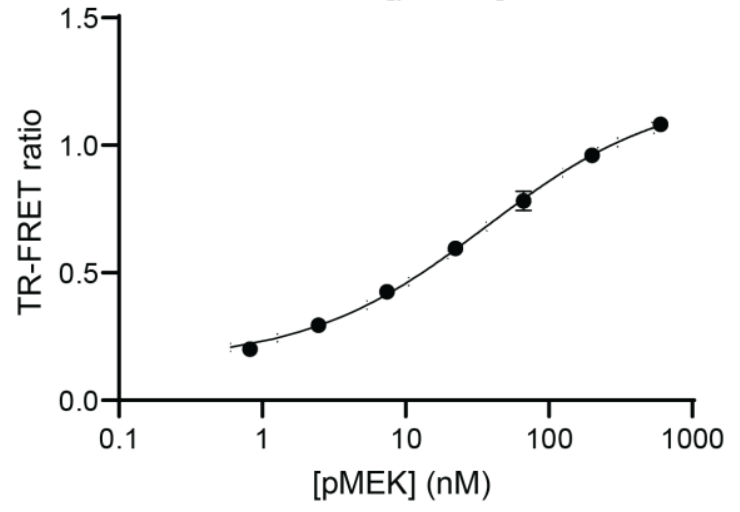

B $40 \mathrm{nM} B R A F^{\mathrm{V} 600 \mathrm{E}}$ timecourse

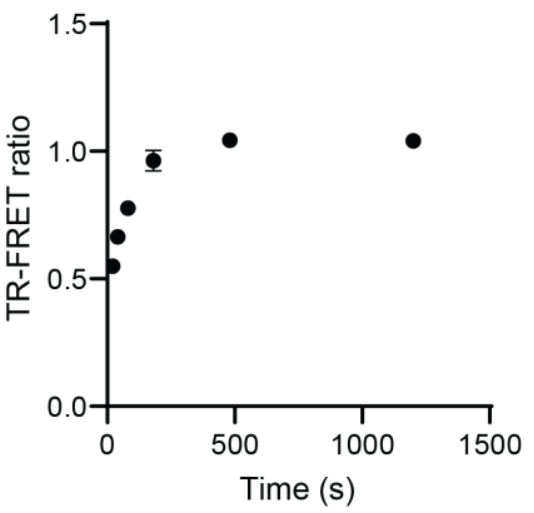

ATM $K_{m}^{\text {app }}$
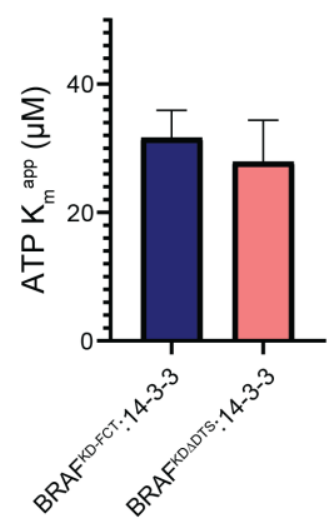

$E$

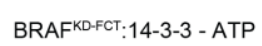

BRAF $^{\text {KD-FCT: }}$ 14-3-3 + ATP

BRAF ${ }^{K D \Delta D T S: 14-3-3 ~-~ A T P ~}$

BRAF $^{\text {KDADTS: }}$ 14-3-3 + ATP
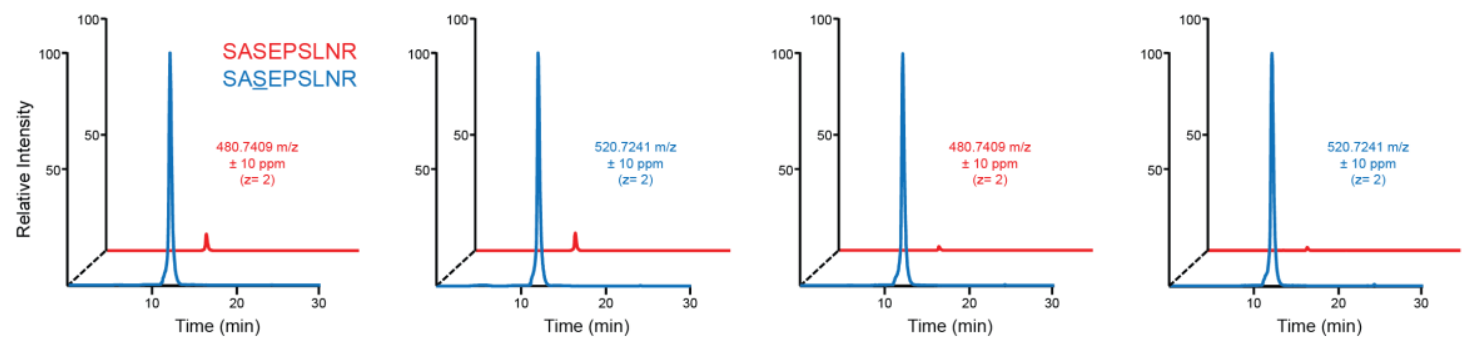

Figure S1. (A) Morrison active site titrations. Error bars represent SD of two technical replicates at two time points $(n=4)$ (B) Representative time course of BRAF V600E phosphorylation of MEK1. Error bars represent SD of two technical replicates $(n=2)$. (C) Representative standard curve of TR-FRET ratio vs [pMEK1], used to produce absolute activity numbers presented in Figure 1B and 1C. Error bars represent SD of two technical replicates $(n=2)$. (D) Summary of BRAF ATP $K_{m}$ from two independent ATP titration experiments, at two time points $(n=8)$. (E) Extracted ion chromatograms comparing relative intensities of peptide containing phosphorylated S729 (blue) and unphosphorylated S729 (red). 
A
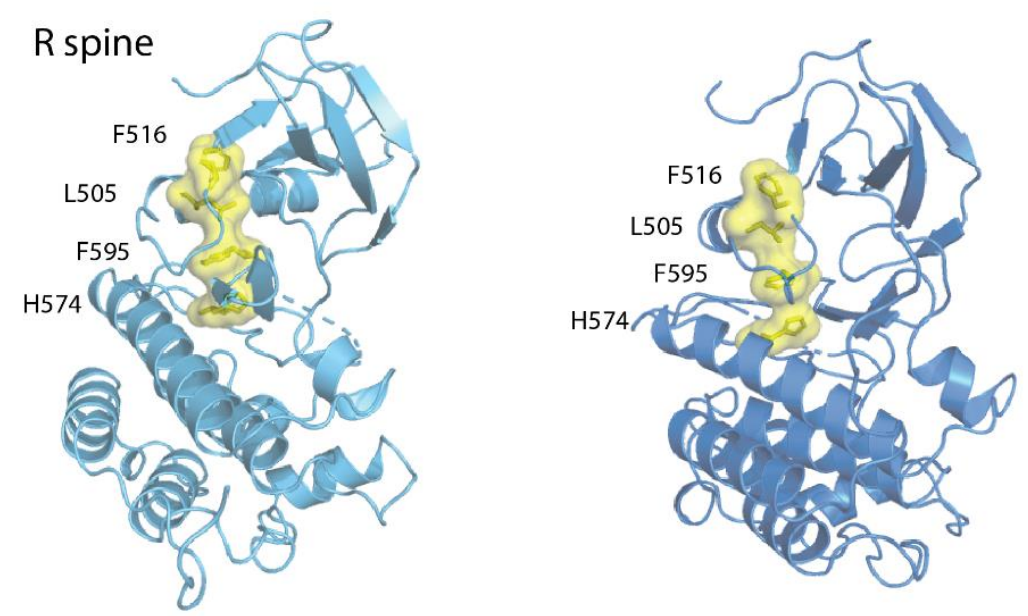

B

C spine
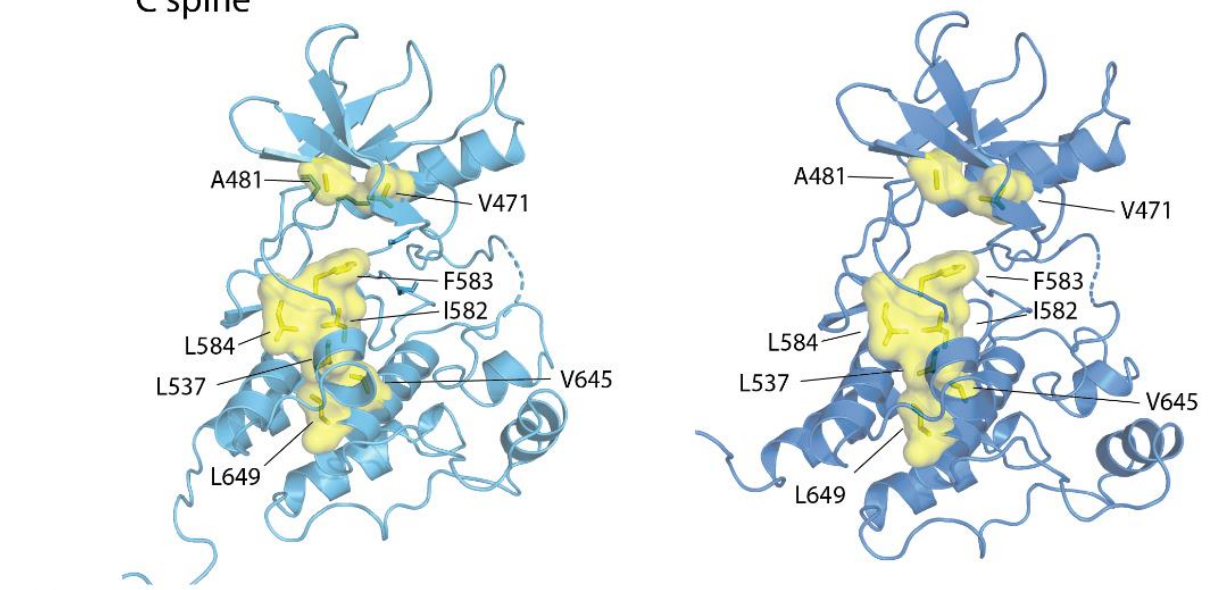

C
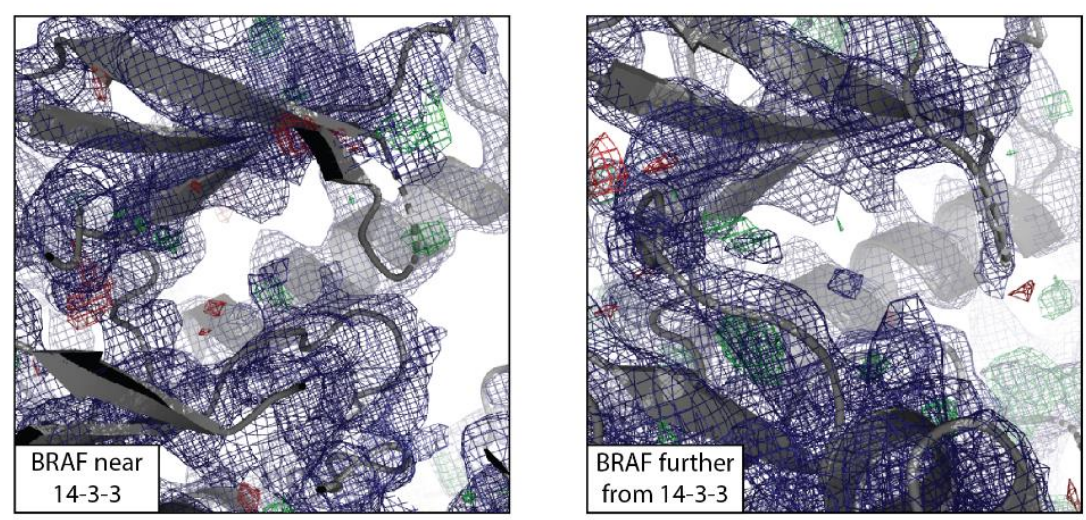

Figure S2. (A) View of assembled R spine (yellow) for the BRAF molecule closer to 14-3-3 (left, light blue) and further from 14-3-3 (right, dark blue). (B) View of $C$ spine (yellow) for BRAF, coloring as per (B). (C) Electron density in ATP binding pocket (2mFo-DFc at $1.0 \sigma$ (blue), mFo-DFc at $\pm 3.0 \sigma$ (green/red)) for BRAF nearer 14-3-3 (left) and BRAF further from 14-3-3 (right). 
A

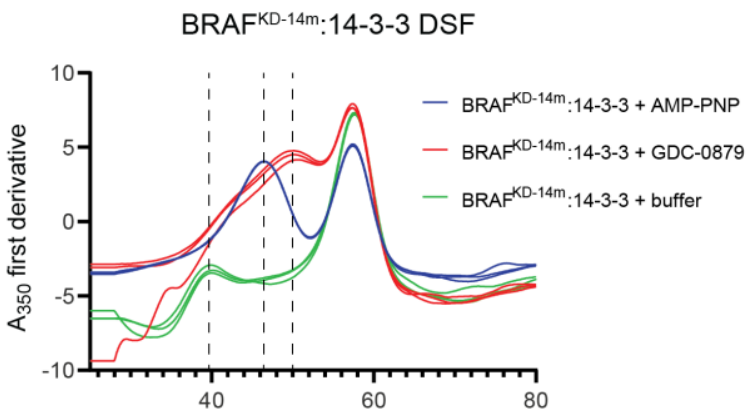

BRAF $^{\text {KDADTS: }}$ 14-3-3 DSF

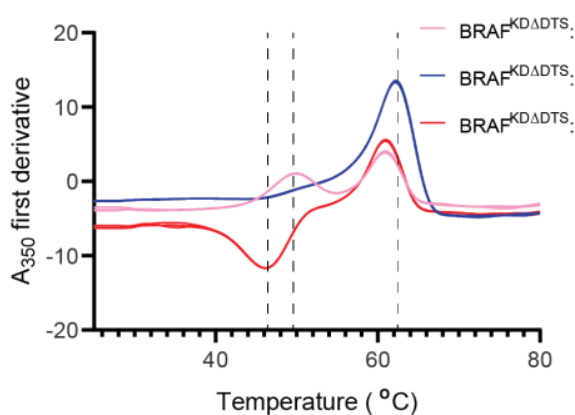

B

BRAF $^{\text {KD-FCT }}: 14-3-3$ raw MST

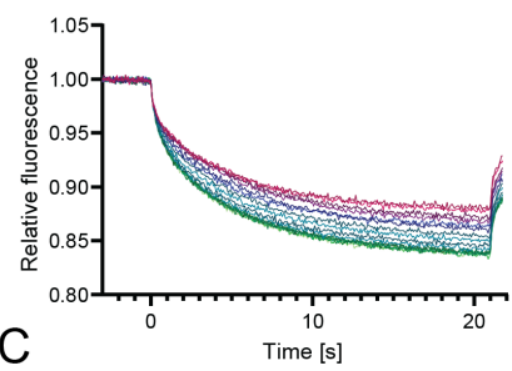

BRAF $^{\text {KD-FCT }}: 14-3-3$ binding curves

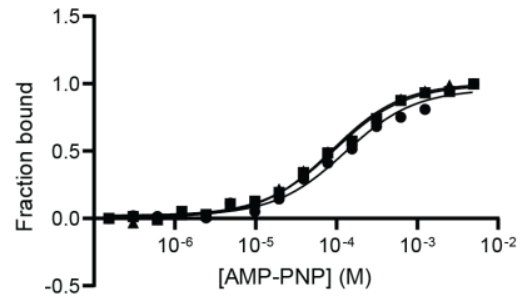

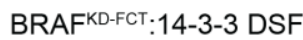

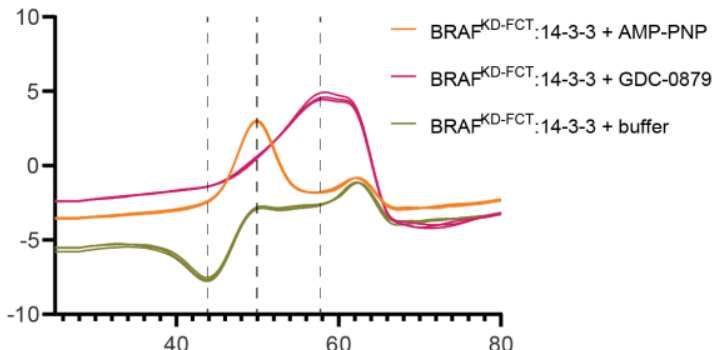

14-3-3 alone DSF

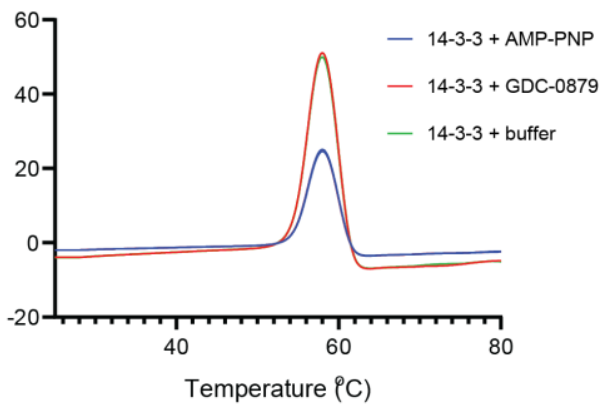

BRAF $^{\text {KD } \Delta D T S}:$ 14-3-3 raw MST

[AMP-PNP] (M)

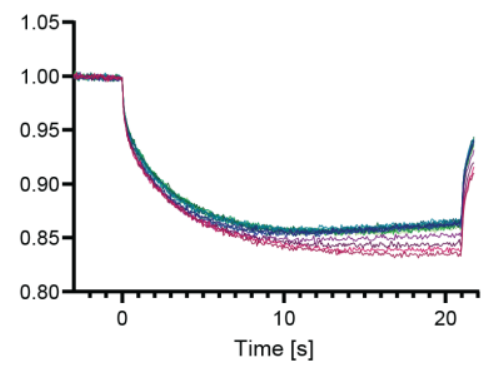

$-0.005$

$-0.0025-9.77 \mathrm{E}-06$

$-0.00125-4.88 \mathrm{E}-06$

$-0.000625-2.44 \mathrm{E}-06$

$-0.000313-1.22 \mathrm{E}-06$

$-0.000156-6.1 \mathrm{E}-07$

$-7.81 \mathrm{E}-05-3.05 \mathrm{E}-07$

$-3.91 \mathrm{E}-05-1.53 \mathrm{E}-07$

Figure S3. (A) Melting curves for BRAF:14-3-3, as the first derivative of $A_{350}$ vs temperature in triplicate for each sample. Top left, top right and bottom left: dashed lines denote first derivative maxima or minima, representing BRAF melting points. Bottom right: melting curves of 14-3-3 alone shows the first derivative maximum at $\sim 60^{\circ} \mathrm{C}$ for each trace represents 14-3-3 melting. (B) Representative raw MST data for AMPPNP binding to BRAF:14-3-3. (C) AMP-PNP binding curves derived from raw MST data. 
HEC1A BRAF-null cells

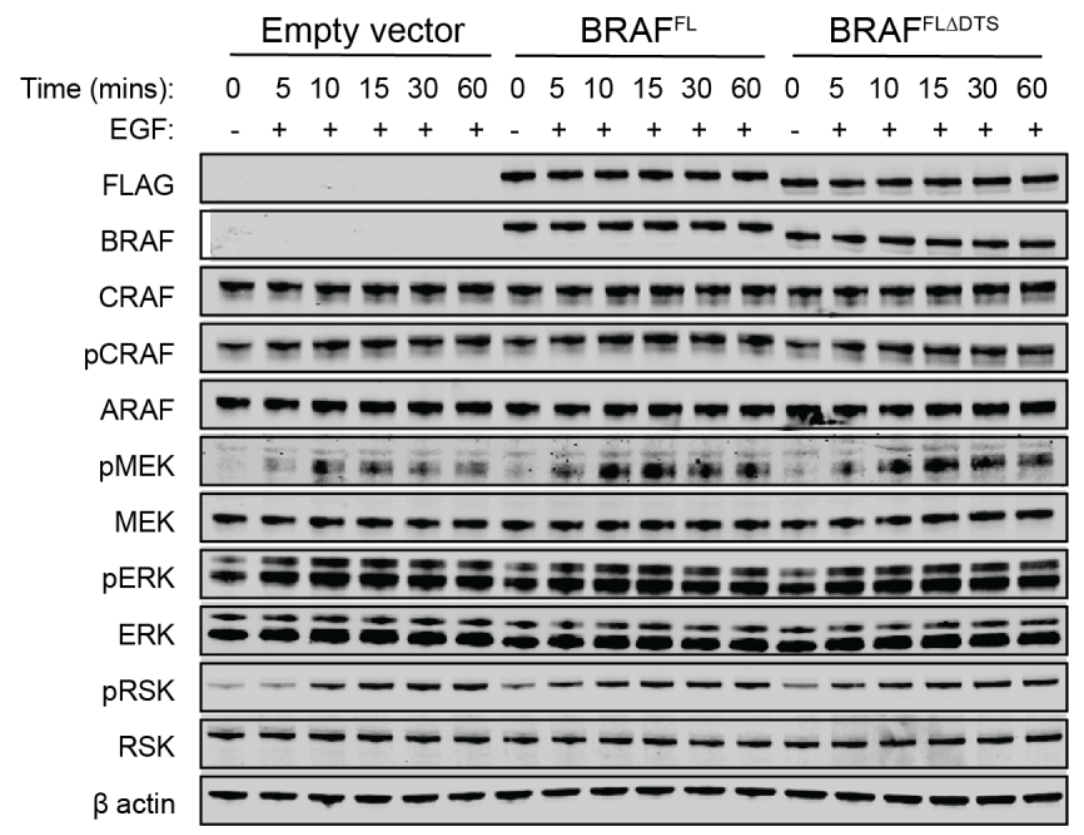

Figure S4. Experimental repeat of western blot from Figure 4 showing elevated pMEK levels for BRAFFL and BRAFFLADTS compared to empty vector control. 
Highlighted: residues within $4.0 \AA$ of DTS

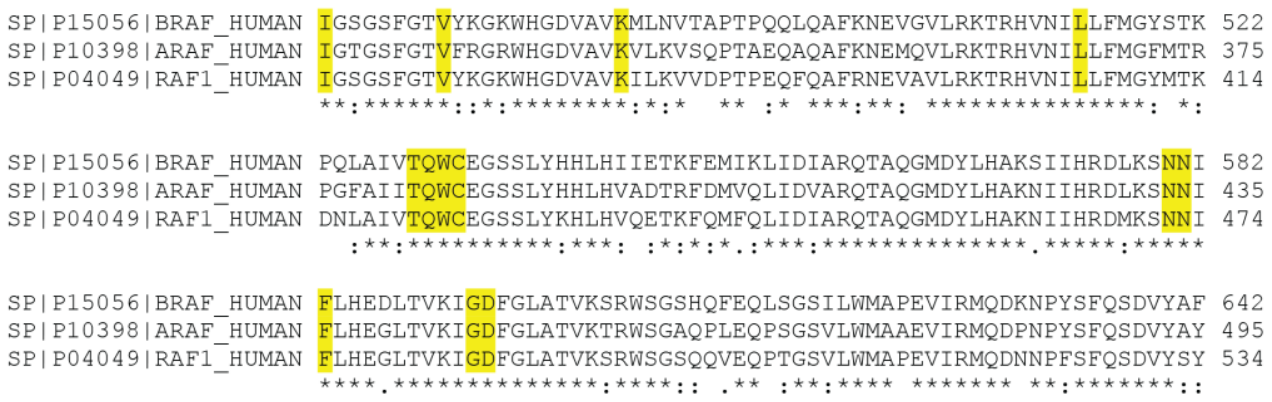

Figure S5. Alignment of residues from BRAF, ARAF and CRAF (RAF1), yellow highlighted residues are within 4.0 Å of DTS in BRAF:14-3-3 Cryo EM structure (PDB: 6UAN). 